\title{
EPR-imaging of magnetic field effects on radiation dose distributions around millimeter-size air cavities
}

To cite this article before publication: Sebastian Höfel et al 2019 Phys. Med. Biol. in press https://doi.org/10.1088/1361-6560/ab325b

\section{Manuscript version: Accepted Manuscript}

Accepted Manuscript is "the version of the article accepted for publication including all changes made as a result of the peer review process, and which may also include the addition to the article by IOP Publishing of a header, an article ID, a cover sheet and/or an 'Accepted

Manuscript' watermark, but excluding any other editing, typesetting or other changes made by IOP Publishing and/or its licensors"

This Accepted Manuscript is @ 2019 Institute of Physics and Engineering in Medicine.

During the embargo period (the 12 month period from the publication of the Version of Record of this article), the Accepted Manuscript is fully protected by copyright and cannot be reused or reposted elsewhere.

As the Version of Record of this article is going to be / has been published on a subscription basis, this Accepted Manuscript is available for reuse under a CC BY-NC-ND 3.0 licence after the 12 month embargo period.

After the embargo period, everyone is permitted to use copy and redistribute this article for non-commercial purposes only, provided that they adhere to all the terms of the licence https://creativecommons.org/licences/by-nc-nd/3.0

Although reasonable endeavours have been taken to obtain all necessary permissions from third parties to include their copyrighted content within this article, their full citation and copyright line may not be present in this Accepted Manuscript version. Before using any content from this article, please refer to the Version of Record on IOPscience once published for full citation and copyright details, as permissions will likely be required. All third party content is fully copyright protected, unless specifically stated otherwise in the figure caption in the Version of Record.

View the article online for updates and enhancements. 


\section{EPR-Imaging of magnetic field effects on}

\section{radiation dose distributions around}

\section{millimeter-size air cavities}

Sebastian Höfel ${ }^{1,2}$, Michael K. Fix ${ }^{3}$, Felix Zwicker², Edmond Sterpin ${ }^{4,5}$, Malte Drescher $^{1}$

${ }^{1}$ Department of Chemistry and Konstanz Research School Chemical Biology, University of Konstanz, Germany

${ }^{2}$ Klinik und Praxis für Strahlentherapie am Klinikum Konstanz, Konstanz, Germany

${ }^{3}$ Division of Medical Radiation Physics and Department of Radiation Oncology, Inselspital, Bern University Hospital and University of Bern, Switzerland

${ }^{4} \mathrm{KU}$ Leuven, Department of Oncology, Laboratory of Experimental Radiotherapy, Leuven, Belgium

${ }^{5}$ Université catholique de Louvain, Center of Molecular Imaging, Radiotherapy and Oncology, Brussels, Belgium

Address all correspondence to:

Prof. Dr. Malte Drescher

Department of Chemistry

University of Konstanz

Box 706, Universitätsstraße 10

78457 Konstanz, Germany

Phone: +49 7531 88-5262

Email: malte.drescher@uni-konstanz.de 


\section{Abstract}

New hybrid radiotherapy treatment systems combining an MRI-scanner with a source of ionizing radiation are being introduced in the clinic. The strong magnetic fields of MRI considerably affect radiation dose distributions, especially at tissue-air interfaces due to the electron return effect (ERE). Experimental investigation of the ERE within a sub-millimeter thick surface layer is still highly challenging.

In the present work, we examine and quantify the magnetic field induced perturbations of dose distributions within a $0.5 \mathrm{~mm}$ layer surrounding millimeter-size air cavities by applying Electron Paramagnetic Resonance Imaging (EPRI).

Air-filled fused quartz tubes (inner diameter 3 or $4 \mathrm{~mm}$ ) mimic small air cavities and serve as model systems. The tubes were irradiated inside a PMMA phantom by a $6 \mathrm{MV}$ photon beam. The irradiations were performed in the presence or absence of a transverse, magnetic field providing a magnetic field strength of 1.0 Tesla. The spatial distributions of radiation induced paramagnetic defects in the quartz tubes were subsequently determined by applying field-swept echo-detected EPRI and were then converted to relative dose distributions.

The transverse magnetic field leads to considerable local dose enhancements and reductions (up to 35\%) with respect to the mean dose within the quartz tubes. The experimentally determined dose distributions are in good quantitative agreement with Monte Carlo radiation transport simulations.

The results of this work demonstrate the feasibility of field-swept echo-detected EPRI to measure magnetic field induced perturbations of dose distributions within a sub-millimeter thick surface layer at the dosimeter-air interface.

Keywords: EPR imaging, dosimetry, radiotherapy, MR-Linac, irradiated quartz 


\section{Introduction}

Modern radiotherapy treatment techniques aim to apply highly conformal radiation dose distributions, i.e. delivering high doses to the tumor cells while sparing surrounding healthy tissue [1]. This approach requires accurate target volume localization and patient positioning prior to each treatment session as well as monitoring of anatomical changes to adapt the treatment if necessary. These requirements may be met by image guided radiotherapy (IGRT) [2].

Magnetic resonance imaging (MRI) has been proposed to serve as imaging modality for IGRT. Hybrid treatment machines combining high energy (MV) photons with an MRI-scanner have been developed [3][6] and are currently introduced in the clinic [7], [8] as MR guided radiotherapy (MRgRT) devices. Compared to X-ray imaging systems, MRI provides superior soft tissue contrast, adds no additional radiation dose to the patient and allows image acquisition in arbitrarily oriented planes. However, MRgRT implies strong, permanent, external magnetic fields (0.35 T [3], 0.6 T [5], 1.0 T [6], $1.5 \mathrm{~T}$ [4]) during irradiation. The orientation of the magnetic field lines is usually either longitudinal (parallel) [5], [6] or transverse (perpendicular) [3]-[6] in relation to the treatment beam. In both setups, the Lorentz force affects the dose distribution by deflecting the trajectory of released electrons that exhibit velocity components perpendicular to the magnetic field lines [9]. Especially for transverse setups, considerable changes in dose distribution may occur - particularly at tissue-air interfaces due to the electron return effect (ERE) [10], [11]. Recently, Rubinstein et al. [12] showed the clinical relevance of the ERE. The authors provided evidence for an increase of radiation induced lung damage in mice that were irradiated in the presence of a transverse $1.5 \mathrm{~T}$ magnetic field. Thus, magnetic field induced perturbations of dose distributions need to be thoroughly investigated, when introducing MRgRT in the clinic.

Numerous theoretical studies have been conducted to examine magnetic field induced perturbations of dose distributions in the context of MRgRT (e.g. [10], [13]- [15]) by using Monte Carlo (MC) radiation transport codes, e.g. Geant4 [16]. Geant4 is a MC simulation toolkit capable of simulating particle transport through matter in the presence of external magnetic fields. It was validated for medical physics applications [17], [18]. Also some commercial dose calculation algorithms were already extended to take the influence of magnetic fields into account [19]. However, experimental investigation and validation of magnetic field induced perturbations of dose distributions is strongly needed.

Recent experimental studies have shown the applicability of EBT3 radiochromic film [20] as well as 3D detectors such as radiochromic plastic, radiochromic gel and polymer gel [21], [22] for the purpose of relative dosimetry in the presence of strong magnetic fields. More recently, Presage ${ }^{\circledR}$ radiochromic plastic was tested for relative dosimetry at dosimeter-air interfaces [23], [24] and showed good agreement with Monte Carlo (MC) radiation transport simulations. However, several authors have shown the limited use of radiochromic film and Presage ${ }^{\circledR}$ dosimeters within the first millimeter from the dosimeter edge due to 
different kinds of artefacts [25], [26], [23]. Besides, these edge artefacts limit the maximum surface-tovolume ratio of such dosimeters and therefore restrict the minimum analyzable detector size.

In the past 60 years Electron Paramagnetic Resonance (EPR) spectroscopy has been widely used to investigate the production, structure and reaction mechanisms of ionizing radiation induced free radicals in various substances [27]. EPR-Imaging (EPRI) is a sensitive method to visualize spatial distributions of paramagnetic centers by utilizing magnetic field gradients for spatial encoding [28]. Eaton and Eaton performed X-Band field-swept echo-detected EPRI to determine electron spin density distributions in irradiated quartz samples [29]. Fused quartz is known to form very stable radicals upon irradiation [30], [31] - the radiation induced $\mathrm{E}^{\prime}$ defects [32]. This is the main class of EPR-active defects in the basic $\mathrm{Si}_{-} \mathrm{O}_{4}$ tetrahedra structure originating from an oxygen vacancy. It is described by an unpaired electron in a nonbonding sp3 hybrid orbital on a silicon [33]- [35]. Impurities in the quartz may lead to additional EPR active defects generated upon irradiation [36]. Besides, two variants of oxygen associated trapped hole centers $(\mathrm{OHC})$ were identified in irradiated fused quartz [37]. Their relative prevalence depends on the $\mathrm{OH}$ content of the specific glass. Moreover, a peroxy radical was found in irradiated fused quartz [38]. Hence, the EPR characteristics of irradiation induced EPR-active defects in quartz may vary depending on the impurity and $\mathrm{OH}$ content as well as on the manufacturing process [30], [33], [39]. In particular, the EPR signal-dose relation is specific to certain types of quartz [39].

The aim of the present work is to examine and quantify the ERE within a sub-millimeter surface layer surrounding millimeter-size air cavities.

For this purpose, air-filled quartz tubes (inner diameter of 3 or $4 \mathrm{~mm}$ ) mimicking cylindrical air cavities are irradiated by a clinical $6 \mathrm{MV}$ photon beam in the presence or absence of a 1.0 T transverse magnetic field. The spatial distribution of irradiation induced paramagnetic defects within the $0.5 \mathrm{~mm}$ thick tube wall are subsequently measured by applying field-swept echo-detected Electron Paramagnetic Resonance Imaging (EPRI). The resulting electron spin density (SD) distributions are converted to dose distributions via a dedicated calibration. For verification, the experimental results are compared to $M C$ simulations using Geant4. 
S Höfel et al. Manuscript submitted to Physics in Medicine and Biology

\section{Methods}

\subsection{Samples and sample holders}

Pieces of ER221/TUB3 and ER221/TUB4 EPR sample tubes (Bruker BioSpin, Billerica, MA, USA) consisting of high quality clear fused quartz with a density of $2.2 \mathrm{~g} / \mathrm{cm}^{3}$ (see Figure $1(\mathrm{a})$ ) were used as quartz samples. TUB3 exhibits an inner diameter (I.D.) of $3 \mathrm{~mm}$ and an outer diameter (O.D.) of $4 \mathrm{~mm}$, whereas TUB4 has an I.D. of $4 \mathrm{~mm}$ and an O.D. of $5 \mathrm{~mm}$. The tubes were cut in pieces of $8 \mathrm{~mm}$ length. A coordinate system $(u, v, w)$ was defined with w pointing along the tubes' symmetry axis (see Figure 1). The unirradiated quartz tube samples are EPR-silent and thus possess no zero dose signal under our experimental conditions.

For irradiation, the samples were placed inside a dedicated composite PMMA phantom (Figure 1(b)). It consists of two outer PMMA plates $(5 \mathrm{~cm} \times 5 \mathrm{~cm} \times 0.5 \mathrm{~cm})$ and an inner PMMA plate $(5 \mathrm{~cm} \times 5 \mathrm{~cm} \times 1 \mathrm{~cm})$. The latter provided a central hole with either $4 \mathrm{~mm}$ or $5 \mathrm{~mm}$ diameter matching the O.D. of TUB3 and TUB4 samples, respectively.

For the EPR-Imaging measurements, the irradiated sample tubes were placed inside Teflon rods with an O.D. of $1 \mathrm{~cm}$ providing centered holes with corresponding diameters (Figure 1(c)).

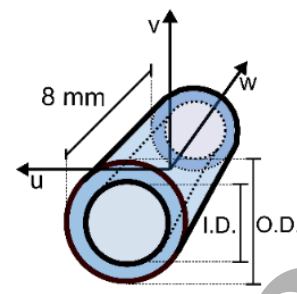

(a)

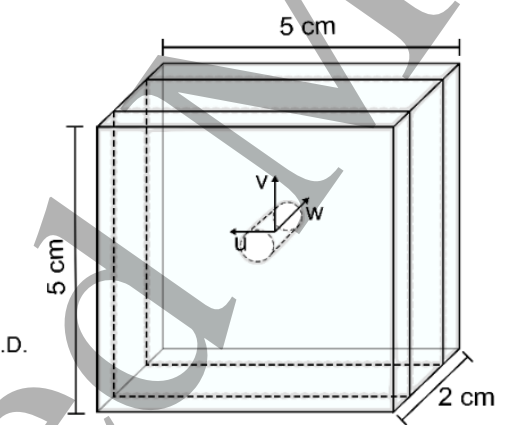

(b)

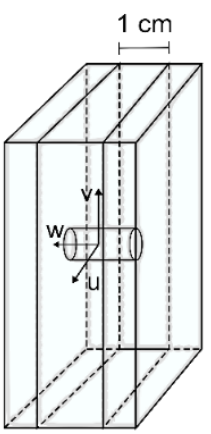

(c)

Figure 1: Quartz tube sample (a) and sample holders $(b, c)$ and the respective orientation of the coordinate system $(u, v, w)$. Definition of the inner and outer diameter (I.D. and O.D.) of the quartz tubes (a). PMMA phantom used for irradiation (b) and sample holder for EPR-Imaging experiment (c). Both provided a central hole of diameter $5 \mathrm{~mm}$ or $4 \mathrm{~mm}$ (depending on the O.D. of the quartz tube).

\subsection{Magnet construction}

A permanent magnet was used to provide a strong, magnetic field of $1.0 \mathrm{~T}$ during irradiation. It consists of two NdFeB permanent block magnets $(10 \mathrm{~cm} \times 10 \mathrm{~cm} \times 3 \mathrm{~cm})$ with magnetization grade N52 (Fa. dogeo $\mathrm{GmbH}$, Waldlaubersheim, Germany) that were mounted on an iron yoke according to Figure 2(a). 


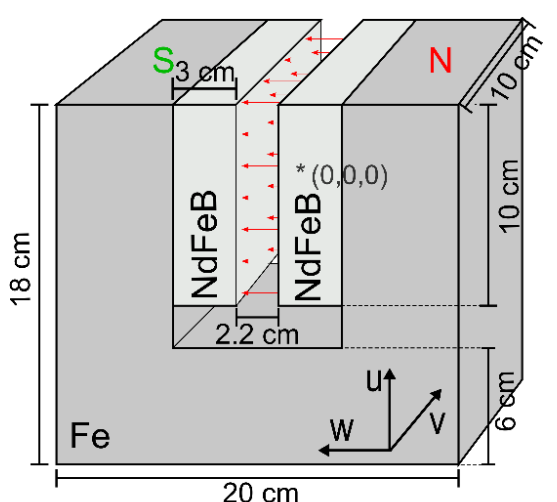

(a)

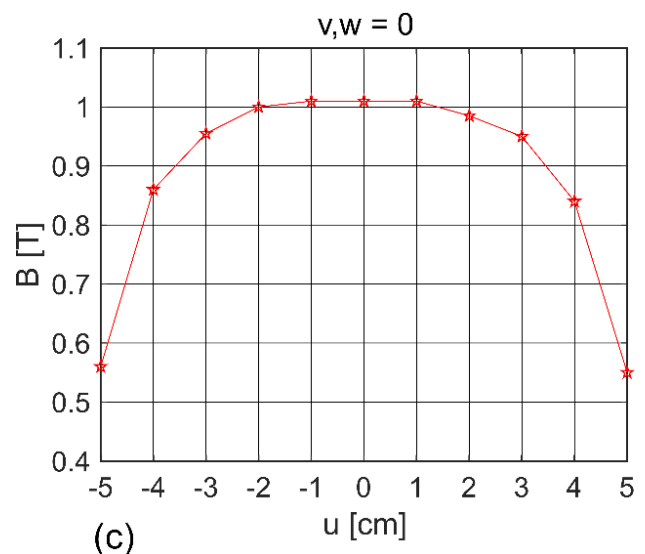

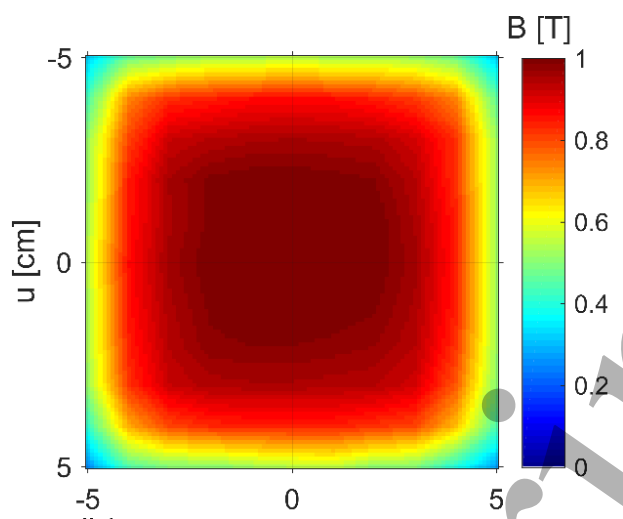

(b)

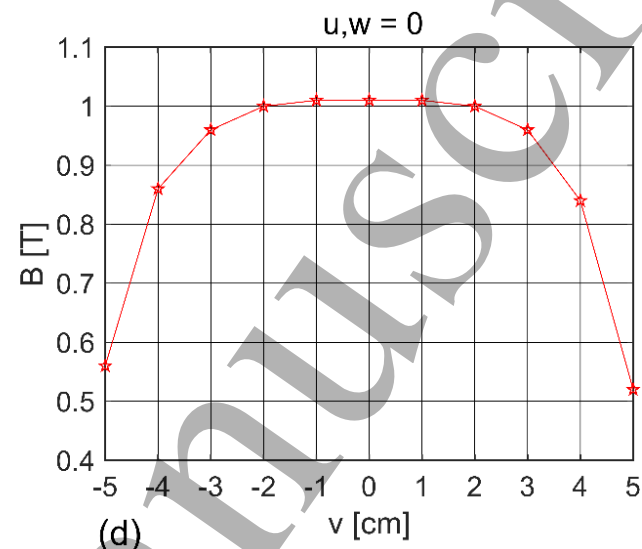

(d)

Figure 2: Permanent magnet construction and experimentally determined magnetic flux density distribution inside the air-gap. Physical dimensions of the magnet construction and relative orientation of the coordinate system (a). The origin is defined as the center of the air-gap. The magnetic field lines inside the air-gap run along the w-direction. Interpolated magnetic field map in the central plane $(u, v, 0)(b)$. Linearly interpolated profiles in $\mathrm{u}$-(c) and $\mathrm{v}$-(d)-direction through the origin. The red asterisks reflect the points of measurement.

The magnetic flux density was measured inside the central plane $(u, v, 0)$ of the air-gap using a teslameter in combination with a hall-sensor (TETelectronics, Schönberg, Germany. Model 3103A and Model 688, respectively). The measurement results are depicted in Figure 2(b)-(d).

Within the central part $(5 \mathrm{~cm} \times 5 \mathrm{~cm})$ of the $(u, v, 0)$ plane the magnetic flux density varies from $0.98 \mathrm{~T}$ at the edges to maximal $1.01 \mathrm{~T}$ in the center.

\subsection{Sample irradiation}

All irradiations were performed using the flattening filter free $6 \mathrm{MV}$ photon beam of a Tomotherapy ${ }^{\circledR} \mathrm{Hi}-$ $\operatorname{Art}^{\circledR}$ (Accuray, Sunnyvale, CA, USA) treatment machine. A static vertical beam with a fixed field size of 9.1 $\mathrm{cm} \times 2.5 \mathrm{~cm}$ (defined at a source-surface distance (SSD) of $85 \mathrm{~cm}$ ) was selected by opening only the central 14 leaves of the binary multileaf collimator (MLC).

The quartz samples were placed inside the corresponding composite PMMA phantom (Figure 1(b)). The interior of the tubes was air-filled. The PMMA phantom was then positioned between the NdFeB permanent magnets such that the center of the quartz tubes was located in the center of the air-gap. The 
magnet construction containing the PMMA phantom was put on top of a water slab phantom and aligned with the treatment beam so that the beam was pointing along the -v-direction and the center of the quartz tube was located on the beam's central axis. The PMMA phantom had a SSD of $62.5 \mathrm{~cm}$ (Figure 3). This setup minimized primary photon radiation through the metal parts of the magnet and still ensured that the PMMA phantom was completely covered by the beam. The samples were irradiated for 45 minutes to doses of $600 \mathrm{~Gy}$.

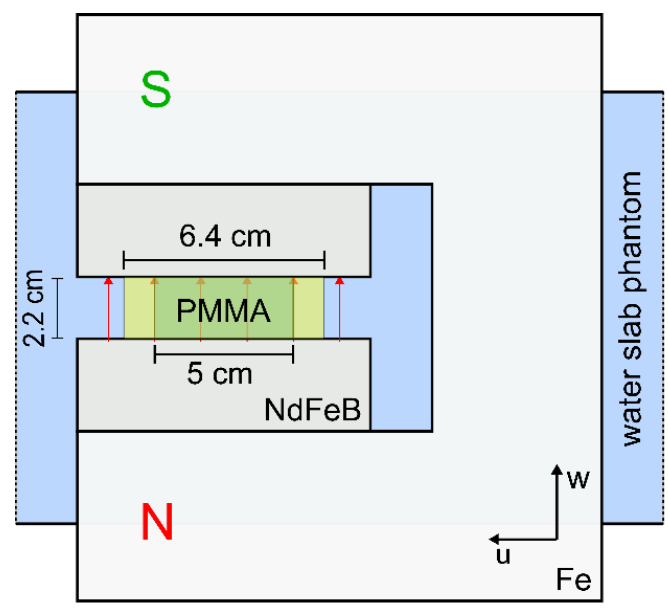

(a)

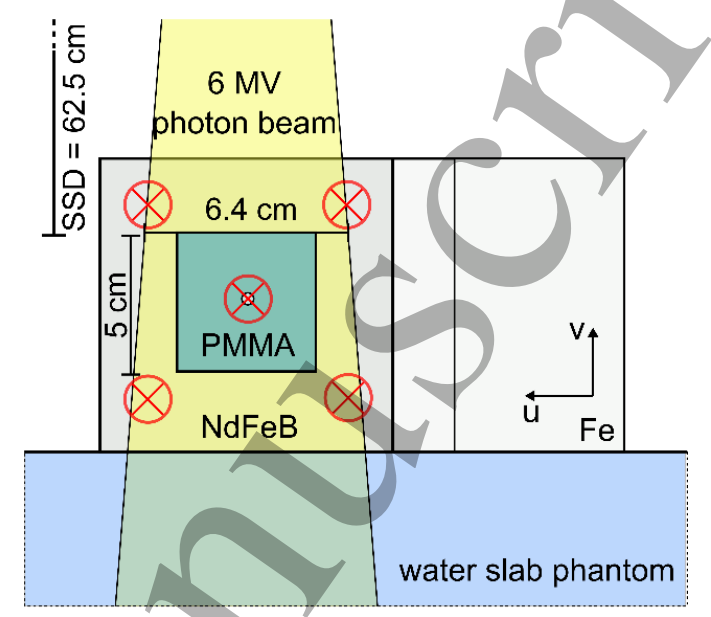

(b)

Figure 3: Irradiation setup. Beam's eye view (a) and transversal section (b) of the setup used for irradiations in the presence of an external magnetic field. The red arrows (a) and crossed circles (b) indicate the direction of the external magnetic field applied during irradiation.

\subsection{EPR experiments}

\subsubsection{EPRI raw data acquisition}

The EPR experiments were conducted at room temperature on a ELEXSYS E580 X-band EPR spectrometer (Bruker BioSpin, Billerica, MA, USA) operating in pulsed mode.

The E580 spectrometer was additionally equipped with a Bruker E 540 GCX2 gradient coil system. The coils provide magnetic field gradient strengths of up to $200 \mathrm{G} / \mathrm{cm}$ in $\mathrm{u}$ - and v-direction (see Figure 4(a)). The $B_{0}$ field lines are parallel to the $u$-axis. By using both gradient coils simultaneously, the gradient of the magnetic field strength $B_{0}$ can be turned into any direction lying in the $u-v-p l a n e$.

For echo detection, the Bruker ER 4108 TMHS imaging resonator was overcoupled ( $Q$ factor was between 100 and 200). The used resonator is a flat cylindrical cavity resonant in the $\mathrm{TM}_{110}$ mode. The resonator profile in v-direction is shown in Figure $4(\mathrm{~b})$. The profile resulted from a one-dimensional imaging experiment of a homogeneous spin density distribution with the magnetic field gradient applied along the v-direction. The samples were positioned in the central plateau region (shaded area in Figure 4(b)), where the profile varies by only a small amount ( $2.5 \%)$ over the dimensions of the sample. In u-direction the resonator profile is nearly flat (0-mode). 


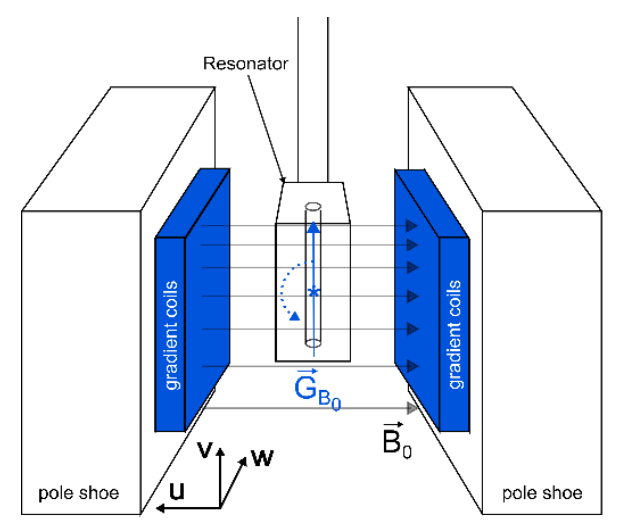

(a)

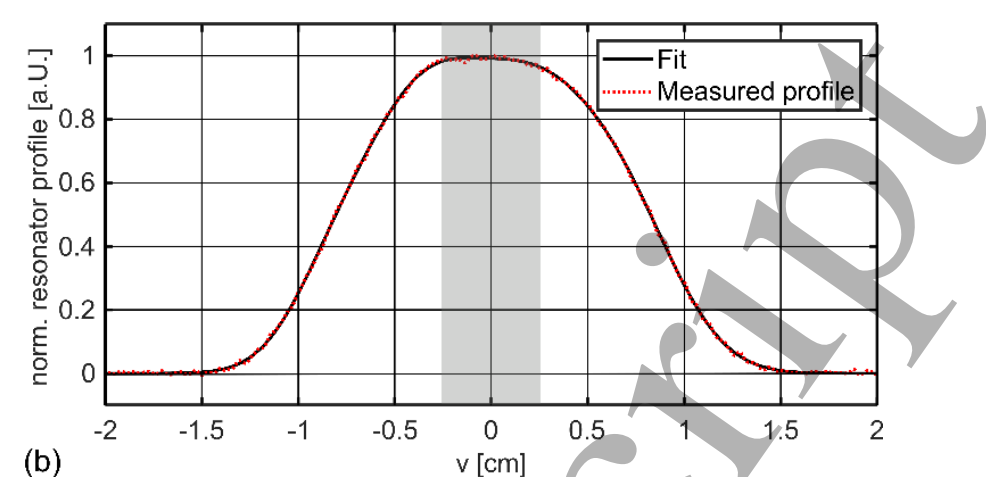

(b)

Figure 4: Schematic sketch of the EPRI setup used for read-out (a). The origin of the coordinate system is indicated by the asterisk. The gradient coil system (blue) is installed between the pole shoes of the electromagnet and the resonator. The blue arrow represents the vector of the magnetic field gradient. The gradient's direction is turned step-wise in the u-v-plane during measurement. Measured and fitted echo intensity profile of the ER 4108 TMHS resonator (b) reflecting the distribution of the square root of the microwave power along the $\mathrm{v}$-direction. The shaded area indicates the plateau region where the samples were placed.

The echo detected field sweeps (EDFS) were recorded by applying a two pulse Hahn-echo sequence ( $\pi / 2-$ $\pi$-echo) to the sample. The sampling resolution of the magnetic field sweep was set to 10 points/Gauss. To minimize spectral line-shape distortions, the excitation bandwidth was kept small by setting the $\pi$ pulse length to $1200 \mathrm{~ns}$. The long phase memory time $\left(\mathrm{T}_{\mathrm{M}} \approx 50 \mu \mathrm{s}\right)$ allowed a respectively long time delay of $2 \mu$ s between the pulses. The spin-echo intensity was recorded by integrating the transient echo signal. Due to the long longitudinal electron spin relaxation time $\left(T_{1} \approx 210 \mu \mathrm{s}\right)$ the EDFS were acquired with a shot repetition time (SRT) of $2000 \mu$ s while 100 shots per data point were averaged. The sweep width was set to $200 \mathrm{G}$ and the sweep time amounted to approximately 10 minutes per EDFS. For imaging, a static gradient strength of $150 \mathrm{G} / \mathrm{cm}$ was applied and 140 EDFS projections were acquired by turning the gradient incrementally by $180^{\circ} / 140$ in the $\mathrm{U}-\mathrm{v}$-plane (Figure 5(a)). Each 2D EPRI measurement ran for 22$24 \mathrm{~h}$ and was performed within three days after irradiation.

For the deconvolution step (Section 2.4.2), an EDFS was recorded after each imaging experiment with the same settings but with the magnetic field gradient turned off (Figure 5(b)). The spectral width of this zerogradient EPR absorption spectrum amounts in total to about $5 \mathrm{G}$ (Figure 5(b)). On the high-field side of the spectrum the signal falls off from $100 \%$ to $10 \%$ of the maximum EPR absorption within $0.8 \mathrm{G}$. 


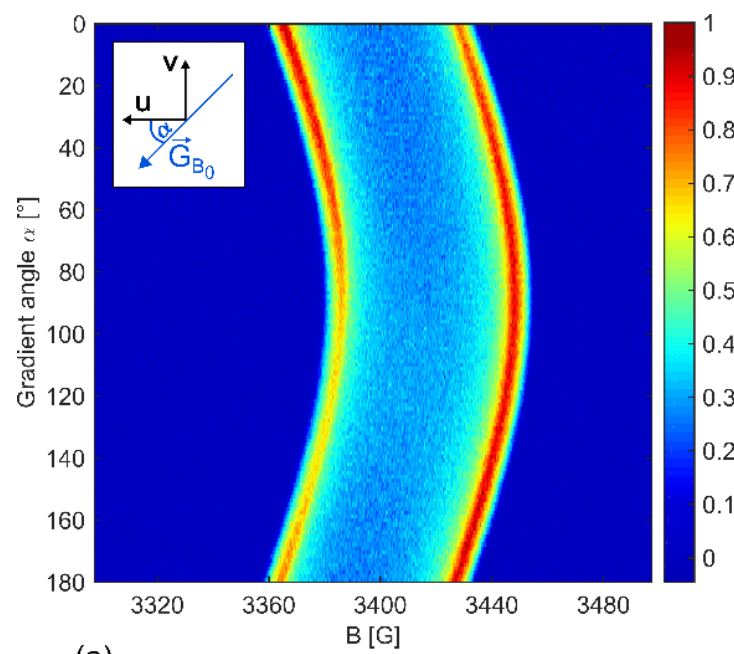

(a)

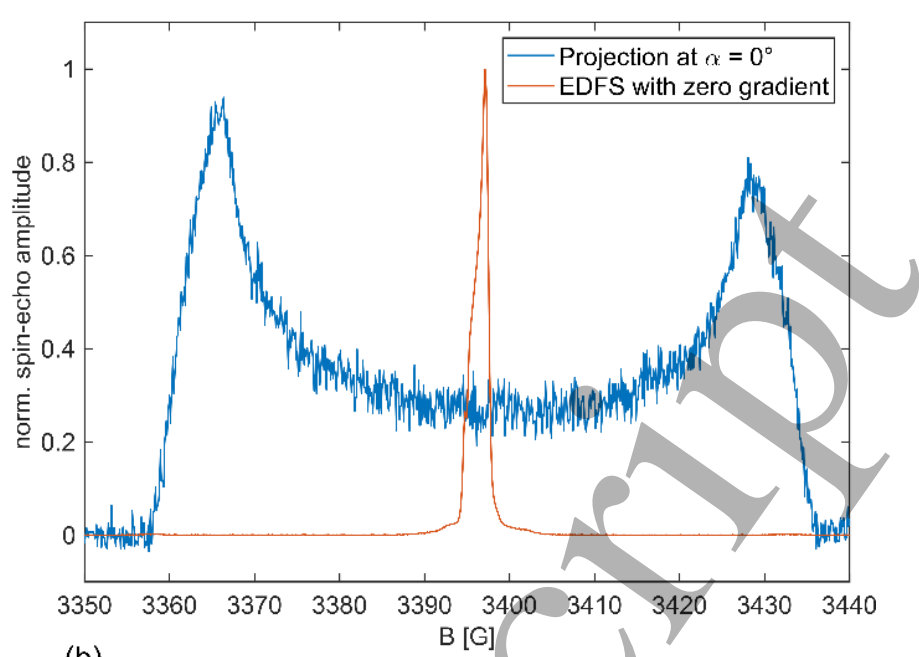

(b)

Figure 5: Exemplary sinogram acquired during the 2D-EPRI measurement of TUB 4 irradiated in the presence of the transverse magnetic field (a). The applied gradient strength was $150 \mathrm{G} / \mathrm{cm}$. The intensity values are normalized to the maximum intensity. The inset defines the projection angle $\alpha$. Central section of the projection acquired under $\alpha=0^{\circ}$ (blue) and of the normalized EPR absorption spectrum (EDFS) recorded without gradient (red) (b).

For the 2D EPRI measurements, the samples were inserted into the appropriate Teflon rod (Figure 1(c)) and positioned such that the center of the tubes were always located at the center of the resonator profile (Figure 4(b)). This position did not perfectly coincide with the center of the magnetic field gradients in vdirection and thus results in a sinusoidal shape of the acquired sinogram (Figure 5(a)).

\subsubsection{Image reconstruction}

Each projection of the resulting sinogram was normalized to its integral intensity to compensate fluctuations in resonator sensitivity during the measurement. Afterwards the projections were deconvolved with the corresponding EDFS spectrum using the regularized filter algorithm of the MATLAB ${ }^{\circledR}$ (MathWorks, Nattick, MA, USA) function deconvreg. During this step, high spectral frequencies (>0.5 1/G) were filtered out. Following deconvolution, the 2D spatial spin distribution was reconstructed using the simultaneous iterative reconstruction technique (SIRT) [40]. For every reconstruction, 100 iterations were applied. Since the EPR absorption spectrum of fused quartz features a steep signal fall off within approximately $0.8 \mathrm{G}$ on the high-field edge of the spectrum (red spectrum in Figure 5(b)) one can estimate a maximum possible imaging resolution of approximately $53 \mu \mathrm{m}$ when a gradient strength of $150 \mathrm{G} / \mathrm{cm}$ is applied. The pixel dimensions for the reconstruction matrix were set to comparable values (40 $\mu$ m (TUB3) and $50 \mu \mathrm{m}(T U B 4))$. These settings fitted well to the frequency filtering associated with the deconvolution step.

After reconstruction, the spin density distribution was corrected for the resonator profiles in $u$ - and vdirection.

For the imaging setup used in this work, the reconstructed images resulting from the EPRI experiments depict the radiation induced relative spin density distribution in the u-v-plane. 


\subsubsection{SD-dose conversion}

For calibration, four additional TUB3 quartz tubes were irradiated by the Tomotherapy beam to doses of 704, 515, 304 and $141 \mathrm{~Gy}$, respectively. The irradiations were performed inside a water-equivalent slab phantom (Accuray, Sunnyvale, CA, USA) and no magnetic field was applied.

Relative SD values were derived from three consecutive one-dimensional EPRI experiments using a static gradient pointing in v-direction. These experiments were performed on composite samples (double stack) where the high dose sample (704Gy) was always measured in combination with one of the lower dose samples. Hence, the high dose sample served as intensity reference for each experiment. The measurement results showing a steplike profile were deconvolved with the EDFS without magnetic field gradient. The mean step intensities were calculated and corrected for the corresponding sample masses. Relative step intensities were then plotted against the corresponding relative dose values. The procedure was performed on day 1 and day 18 after irradiation in order to check the reproducibility of this approach. A SD-dose calibration curve was obtained by applying a polynomial fit to the data.

\subsection{Monte Carlo simulation}

MC Simulations were performed with version 10.3 of the Geant4 toolkit. The low-energy electromagnetic models for electron and photon interactions were activated by selecting G4EmPenelopePhysics as physics list. The lower energy threshold limit for production of secondary particles was decreased to $100 \mathrm{eV}$ and the range cut for the production of secondary electrons and photons was set to $0.05 \mathrm{~mm}$ according to the minimum dimension of the voxels of the scoring volume (see below).

The simulation geometry was defined following the irradiation setup shown in Figure 3. For the NdFeB magnets an alloy material according to the elemental composition with a density of $7.6 \mathrm{~g} / \mathrm{cm}^{3}$ was created.

In the simulations considering a magnetic field during irradiation a global uniform magnetic field of 1.0 Tesla along the $w$-direction was assumed. The step size limitations ( RRoverRange $=0.003$, finalRange $=$ $1 \mathrm{~nm}$ ), the tracking parameters in magnetic fields (DeltaChord $=1 \mu \mathrm{m}$, MinimumEpsilonStep $=5 \mathrm{e}-5$, MaximumEpsilonStep $=5 \mathrm{e}-5$, Deltalntersection $=1 \mu \mathrm{m}$, DeltaOneStep $=1 \mu \mathrm{m}$ ) as well as the multiple scattering parameters (RangeFactor $=0.01$, GeomFactor $=2.5$ and $s k i n=2$ ) were set according to Lee et al. [41]. A time efficient $4^{\text {th }}$ order Runge-Kutte-Nystrom stepper (G4NystromRK4) was activated to integrate the equation of motion of a particle inside a homogeneous magnetic field. To define the Tomotherapy radiation beam, a validated phase-space file was implemented [42], [43]. The position of the dose scoring volume with dimensions of $8 \mathrm{~mm} \times 8 \mathrm{~mm} \times 8 \mathrm{~mm}$ was defined such that the center of the scoring volume coincided with the quartz tube axis. The volume was divided in 160 bins along the $u$ and v-direction.

The statistical uncertainties resulting from 20 independent runs were analyzed. The relative standard errors for the mean dose values inside the quartz tubes were below $2 \%$. 


\section{Results}

2D EPR imaging experiments were performed on three irradiated quartz samples. Two quartz tubes with inner diameters of $3 \mathrm{~mm}$ (TUB3) and $4 \mathrm{~mm}$ (TUB4), respectively, were irradiated in the presence of a transverse magnetic field of $1.0 \mathrm{~T}$ provided by a permanent magnet (section 2.2). As a control experiment, one additional TUB4 sample was irradiated without external magnetic field by using a non-magnetic dummy iron yoke that had the same dimensions as the magnet but provided no magnetic field.

The imaging experiments were performed in pulse mode since the known long spin relaxation times of the irradiation induced defects in fused quartz samples make it difficult to obtain unsaturated CW-EPR spectra free of passage effects [44]. In pulse mode, sequences of short (ns- $\mu \mathrm{s})$, high power microwave pulses are applied to the sample and spin-echo intensities are detected. The EPR absorption spectrum is detected by echo detected field sweeps (EDFS), i.e. recording spin-echo intensities while the magnetic field strength $B_{0}$ is swept. In contrast to the time-domain (Fourier-transform) spin-echo detected EPRI method, this approach is not limited to small gradient strengths [28]. EDFS measurements were measured in combination with magnetic field gradients to perform imaging experiments.

When applying magnetic field gradients to a sample the spatial information of spin density distribution is transformed to spectral information. In case that the spectral shape is constant throughout the sample, the EPR spectrum acquired under a certain gradient angle represents a convolution of the radon transform of the spin density under this angle with the spectral line shape [40].

It was assumed that the spectral line shape is constant throughout the irradiated sample and that it is equivalent to the spectrum given by the EDFS recorded at zero gradient strength.

In control experiments, no relevant dose-dependencies of crucial EPR characteristics (temporal stability, spectral shape and spin relaxation times) were observed in the dose range between 141 and $704 \mathrm{~Gy}$.

\subsection{SD-Dose dependency}

In general, EPRI reveals images of relative SD distribution. To convert the reconstructed SD distribution to a dose distribution the SD-dose dependency was investigated. For this purpose, the 'calibration samples' were used (section 2.4.3) to determine a calibration function that converts relative SD values to relative dose values. The post processed (section 2.4.3) results are shown in Figure 6. 


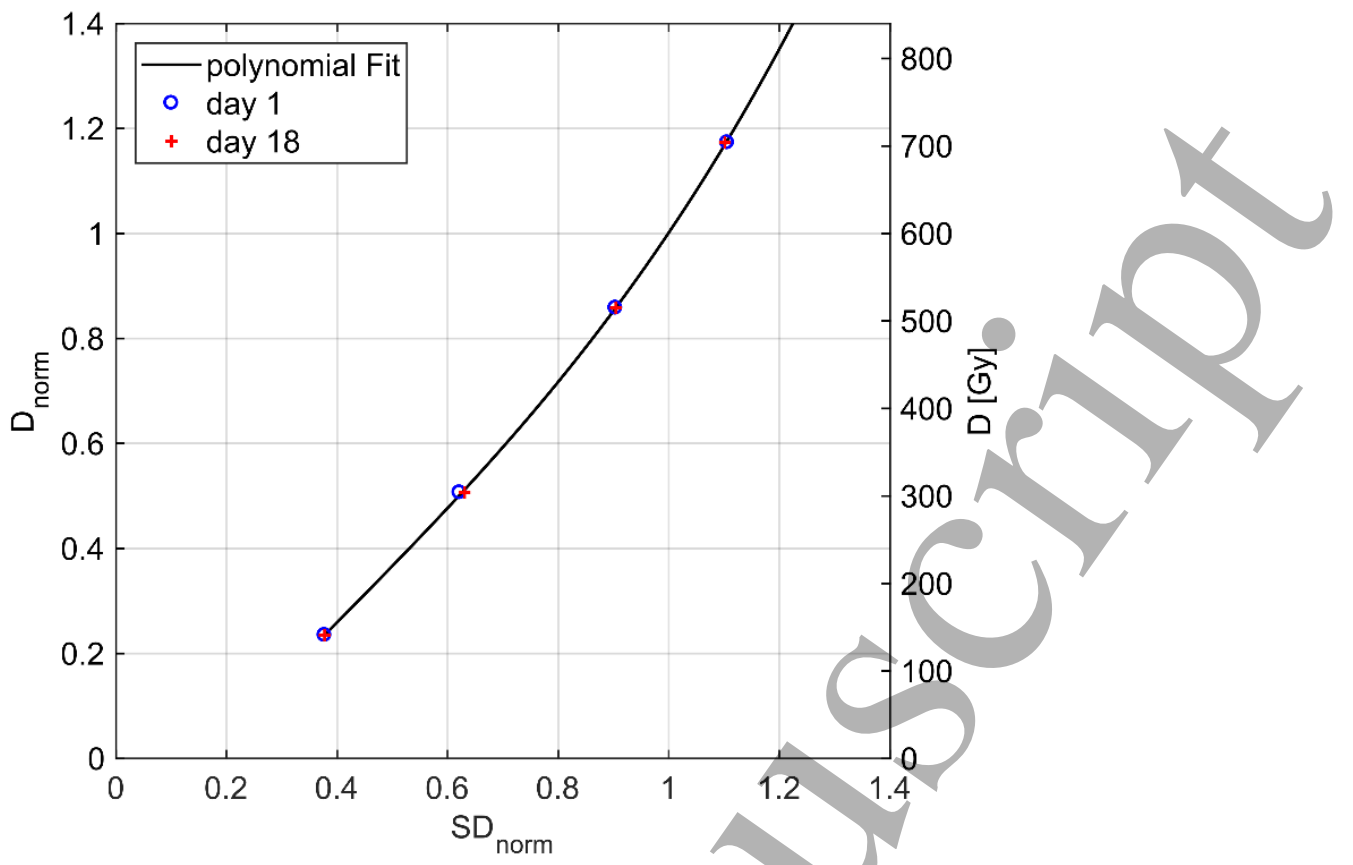

Figure 6: Dependency of relative spin densities ( $S D_{\text {norm }}$ ) derived from 1D-EPRI measurements on the dose $\left(D_{\text {norm }}\right)$ normalized to an irradiation dose of $600 \mathrm{~Gy}$. The results for day 1 and day 18 post irradiation are shown. The SD-dose dependency is described by a 3rd order polynomial fit.

The relative SD and relative dose values are normalized to an irradiation dose of $600 \mathrm{~Gy}$. The relative standard errors for the derived relative SD values were below $1.5 \%$.

When comparing the results from both measurement days, the derived relative SD values are identical within this uncertainty. This indicates that signal fading (approximately $10 \%$ ) within 18 days post irradiation does not affect the SD-dose calibration.

For the investigated dose range, the relative SD values show a slightly non-linear dependency with respect to the relative dose values. Therefore, a 3rd order polynomial function was fitted to the data. In the following this polynomial fit is applied to the EPRI experiments to convert relative SD distributions to relative dose distributions.

\subsection{EPRI and $M C$ simulation results}

The 2D EPRI raw data was processed following the procedure given in section 2.4. Each pixel of the reconstructed $2 \mathrm{D}$ image represents the electron spin density integrated along the quartz tube axis. Afterwards, the reconstructed relative SD distribution was converted to a relative dose distribution by applying the above derived SD-dose calibration curve (section 3.1). The results for all 2D EPRI measurements are shown in Figure 7 (left side). For comparison, the corresponding MC simulation results are shown on the right side. The radiation beam enters the quartz sample from above, i.e. the beam direction points along the $-v$-direction. The magnetic field during irradiation is to be imagined pointing into the image plane (along the w-direction, see schematic sketch in Figure 7). 
S Höfel et al. Manuscript submitted to Physics in Medicine and Biology

The magnetic field strength applied during irradiation affects the trajectories of the released, secondary particles (mainly electrons). Small field variations $(<2 \%)$ within the central part $(5 \mathrm{~cm} \times 5 \mathrm{~cm})$ of the magnet (Figure 2) have a negligible impact as verified by corresponding MC simulations (data not shown).

By analyzing the simulated dose distributions (Figure 7, right side) prior to normalization, it was noticed that the mean dose to the whole quartz sample did not differ by more than $1.2 \%$ between those three simulations. To compare the relative dose distributions, the dose colormaps were normalized to the corresponding mean dose value.

Likewise, the reconstructed relative SD values were normalized to the mean value inside the quartz and then the relative SD distributions were converted to dose distributions using the calibration curve in Figure 6 (normalized to $600 \mathrm{~Gy}$ ). Hence, relative dose values with respect to the mean dose value were directly obtained.

The calibration curve is valid down to $141 \mathrm{~Gy}$ and may not be applied at lower doses. Hence, dose values below $25 \%$ of the mean dose (600 Gy) are omitted by the color scales in Figure 7.

The EPRI result for the TUB4 sample irradiated with no magnetic field applied shows a slight inhomogeneity in dose distribution. A maximum of dose deposition is observed at the upper tube side where the radiation beam entered the sample. This inhomogeneity is also seen in the MC simulations.

By comparing the $0 \mathrm{~T}$ with the $1 \mathrm{~T}$ results for the TUB4 samples one can observe magnetic field induced perturbations of the dose distribution inside the quartz. The dose homogeneity is substantially different compared to the $0 \mathrm{~T}$ case. This effect is seen for both the measurements and MC simulations. The measured dose distributions obtained for the TUB3 sample shows similar dose enhancements and reductions but to a reduced extent as the TUB4 (1 T) sample.

As an artefact of image reconstruction the edges of the quartz tube wall are blurred. This is mainly due to the limited number of acquired projection angles and the high frequency filtering applied during regularized deconvolution (section 2.4.2). The apparent fluctuations in the reconstructed dose distribution are due to the noise already present in the raw data (Figure 5) and due to the SIRT reconstruction technique. SIRT has advantages over filtered back projection regarding image resolution when dealing with noisy input data [40]. In that cases, however, SIRT is prone to cause salt and pepper noise. 

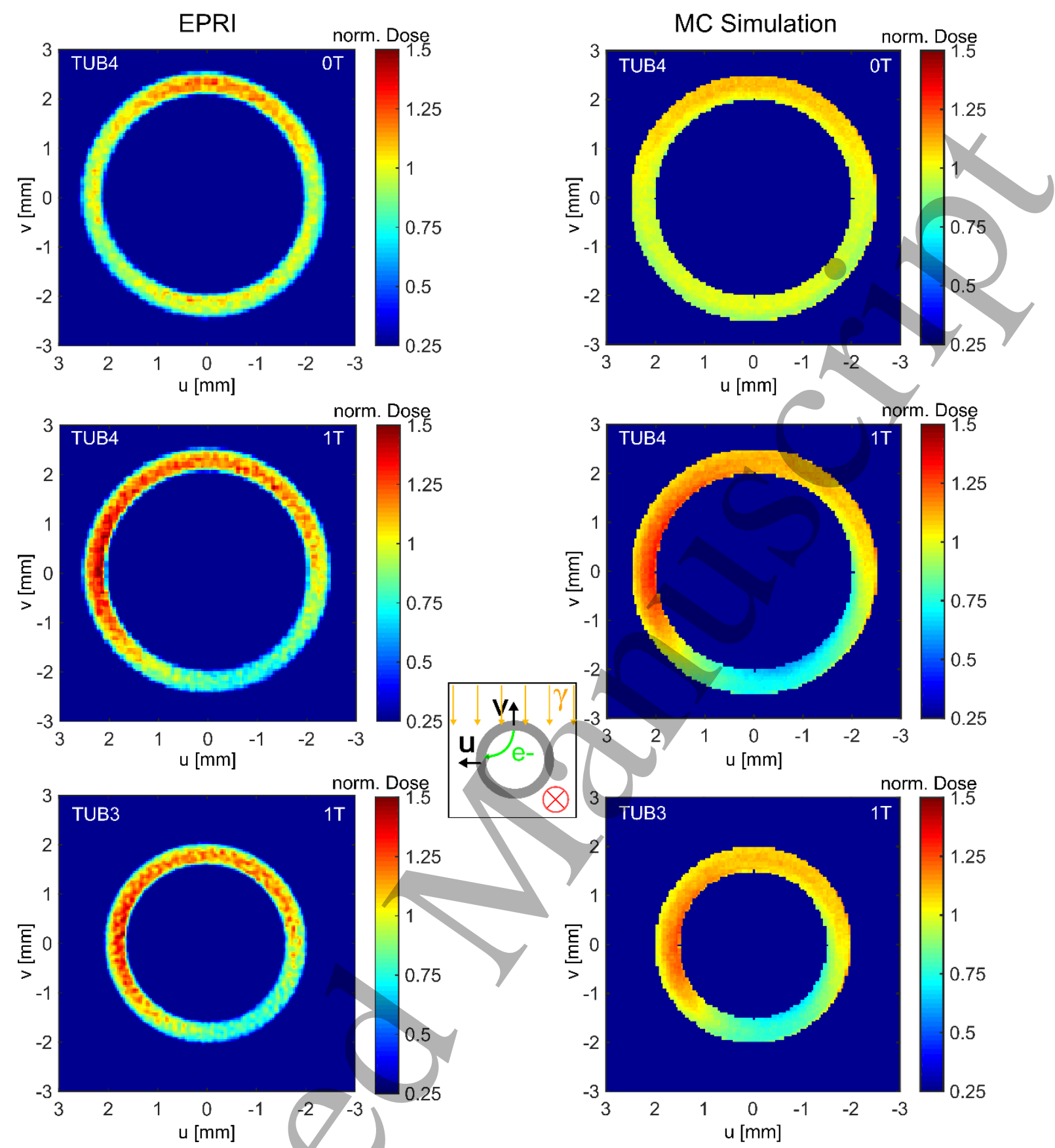

Figure 7: Comparison of reconstructed normalized dose distributions (left) and the corresponding MC simulation results (right) for the sample TUB4 irradiated in no external magnetic field (top), TUB4 (middle) and TUB3 (bottom) irradiated in the presence of a $1.0 \mathrm{~T}$ magnetic field. The reconstructed relative SD values were converted to relative dose values as described in the text. The dose values of the MCSimulation are normalized to the mean dose in the quartz tube. The inserted sketch illustrates the effect of the transverse magnetic field on the trajectories of secondary electrons entering the air cavity along the beam direction.

In order to improve the signal-to-noise ratio (SNR) and to further examine the perturbation of the dose distribution over the quartz tubes, a polar coordinate transformation was applied to the 2D-dose distributions obtained in the presence of the $1.0 \mathrm{~T}$ magnetic field. The dose values were radially averaged across the quartz tube wall and the statistical uncertainties (standard error) of the mean values were 
determined. The polar angle was defined in relation to the v-axis. In the graphical representations given in Figure 7 the polar angle increases clockwise. The resulting representation of the dose distributions are shown in Figure 8.
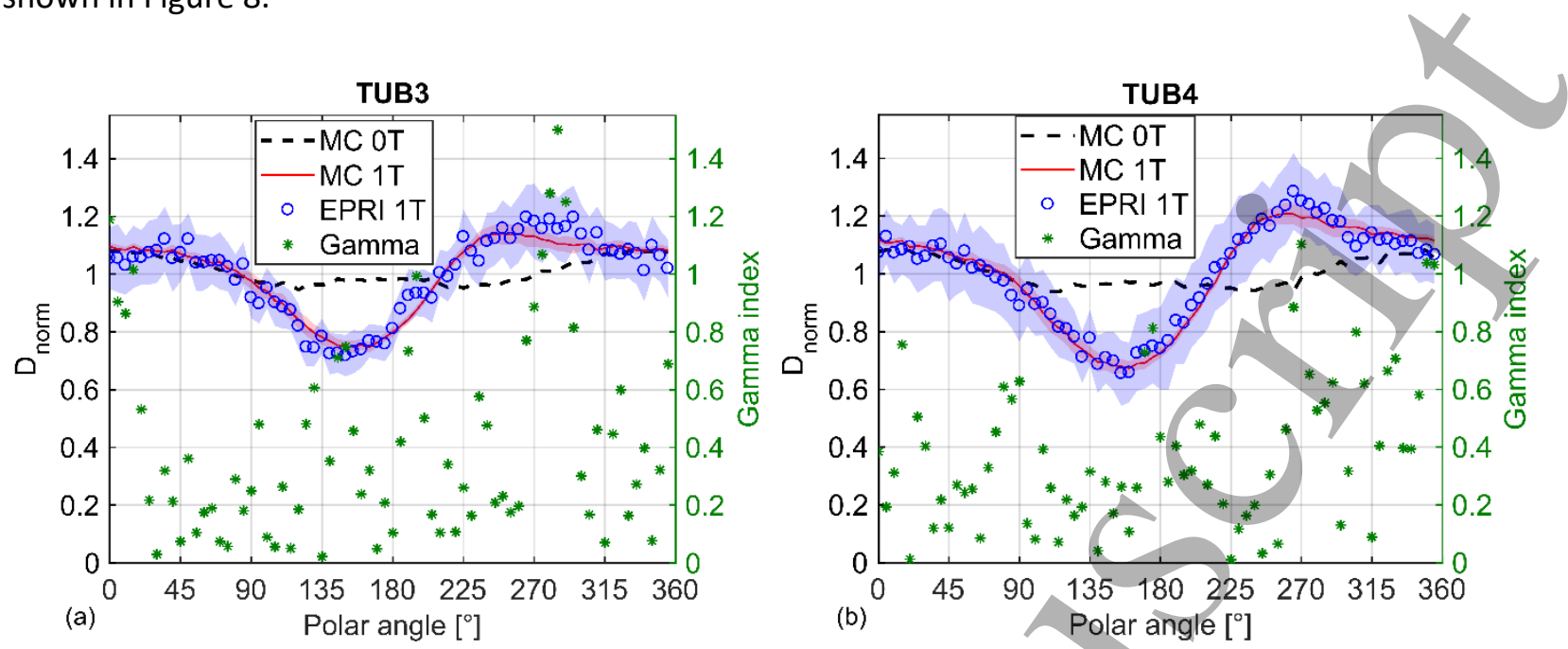

Figure 8: Dose distributions over the quartz sample tubes TUB3 (a) and TUB4 (b) in polar coordinates and results of the gamma evaluation ( $3 \% / 10^{\circ}$ criterion). Measured and simulated dose distributions are shown for the presence of a $1.0 \mathrm{~T}$ external magnetic field. For comparison purposés, the corresponding simulated dose distribution obtained for zero magnetic field is displayed. The displayed confidence bounds are the standard errors of the mean dose values resulting from radial averaging.

For the samples irradiated in the magnetic field of 1.0 T dose enhancements of up to $20 \%$ (TUB3) and $28 \%$ (TUB4) with respect to the mean dose were measured. The polar angle of maximum dose deposition for TUB3 and TUB4 is between $250^{\circ}$ and $280^{\circ}$. On the other hand, substantial dose reduction of $28 \%$ (TUB3) and $35 \%$ (TUB4) at a polar angle between $140^{\circ}-160^{\circ}$ arise. The dose enhancement and reduction is thus less pronounced for the TUB3 sample. The measured dose distributions are in good agreement with the MC simulation results as shown by the confidence bounds overlap. A gamma analysis according to Low et al. [45] was performed to quantitatively compare the measured and simulated circular dose distributions. The simulated data served as reference and a global dose difference tolerance of $3 \%$ of the simulated dose maximum was used. For a 3\%/10 global gamma criterion, $96 \%$ (TUB4) of the data points obtained via EPRI (Figure 8 (b)) yield a gamma value below 1 . The same gamma analysis for the TUB3 sample (Figure 8 (a)) yields a gamma passing percentage of $92 \%$. 


\section{Discussion}

In the current work, field-swept echo-detected EPRI was applied for the first time to determine relative dose distributions around small, cylindrical air cavities (I.D. of 3 or $4 \mathrm{~mm}$ ) in the presence or absence of a transverse, magnetic field of 1.0 T - a highly topical issue regarding the introduction of MRgRT in the clinic. The experimentally derived dose distributions are quantitatively in good agreement with the corresponding MC simulation results. These findings support the feasibility of field-swept echo-detected EPRI to determine magnetic field induced perturbations of dose distributions and provide experimental evidence for the ERE occuring at millimeter-size air-cavities.

So far, only few studies addressed dosimetric issues in the field of medical radiation science by applying EPRI. For example, relative dose distributions in human bone samples as well as in hydroxyapatite phantoms were reconstructed using X-Band Continuous Wave (CW) EPRI [46]. The samples were irradiated in the vicinity of gamma radiation sources typically used in brachytherapy applications (i.e. lodine-125, Iridium-192). Vanea et al. [47] used L-Band CW-EPRI and solid lithium formate to depict the steep dose gradients around brachytherapy sources. Kolbun et al. [48] measured the dose distribution in lithium formate and ammonium formate when irradiated in the vicinity of an Ir-192 source.

The present work focused on the dosimetric consequences of the ERE within a $0.5 \mathrm{~mm}$ surface layer surrounding millimeter-size air cavities. To date, there has been no experimental work reliably quantifying the ERE for such situations. This is presumably due to the fact that alternative dosimeters such as rodiochromic films or plastics typically show artefacts within the first millimeter from the dosimeter edge [25], [26], [23] and are therefore also restricted in their minimum size. On the other hand, the maximum possible sample size of on EPRI dosimeter is physically restricted by the resonator's profile. Roughly speaking, X-Band EPRI is thus limited to a maximum samples size of approximately $1 \mathrm{~cm}$. We therefore propose EPRI to be a complementary method to other, well-established relative dosimetry techniques.

The experimental results of this work clearly depict a substantial influence of the external magnetic field on the dose distribution within the sample material surrounding the air cavity. The dose distributions show dose maxima at a polar angle between $250^{\circ}$ and $280^{\circ}$ as well as dose reductions at a polar angle between $140^{\circ}-160^{\circ}$.

For a cursory explanation of the magnetic field induced dose perturbations, one can estimate the radius of curvature in vacuum for the/trajectories of electrons released during irradiation. According to Raaijmakers et al. [10], reasonable assumptions for calculating the mean electron energy are that the mean photon energy is given by one third of the accelerator voltage times the elementary charge and that the mean kinetic energy of the electron spectrum is approximately one quarter of the average photon energy. For a $6 \mathrm{MV}$ photon beam the mean electron energy is thus estimated to about $0.5 \mathrm{MeV}$. Given a magnetic field strength of $1.0 \mathrm{~T}$ the radius of curvature for an electron with a kinetic energy of $0.5 \mathrm{MeV}$ is approximately $2.9 \mathrm{~mm}$. This radius of curvature is in the same order of magnitude as the inner diameters 
( 3 and $4 \mathrm{~mm}$ ) of the air-filled cavities studied in this work. Therfore, one had to expect perturbations of dose distributions in the quartz sample tube surrounding the air cavity due to the ERE: Electrons entering the air cavity start to spiral clockwise (sketch in Figure 7) due to the Lorentz force. As a result, dose enhancements appear on the left side (with respect to Figure 7) of the tube wall for the samples irradiated in an external magnetic field. This effect is mainly caused by electrons with a kinetic energy lower than the estimated mean kinetic energy of $0.5 \mathrm{MeV}$.

The previous consideration and the results shown in Figure 7 and 8 indicate that the dosimetric impact of the ERE occuring at millimeter-size air cavities decreases with decreasing cavity diameter. Besides, the results provide experimental evidence that for cylindrical air cavities with a diameter of 3-4 $\mathrm{mm}$ the dose perturbations induced by the ERE are not fully compensated by applying parallel opposed beam configurations. A similar conclusion was already drawn from previous MC simulations for an air tube with a diameter of $2 \mathrm{~cm}[10]$.

Prior to the imaging experiments, a SD-dose calibration curve was determined for the quartz samples used in this work. This step was necessary, since the dose response highly depends on the specific type of quartz glass [39]. It is further known from literature that different types of $E^{\prime}$ defects may be generated upon irradiation [49], [50]. It was also reported that for the dose range investigated in this work, defects are generated rather from precursors [39], [51] than from direct matrix activation.

For the quartz samples and dose range investigated in this work, a monotonous, slightly non-linear dose dependence of the reconstructed SD was observed (Figure 6). The slight non-linear SD-dose dependency can be explained by Galeener's phenomenological creation and activation model [39]: Irradiation leads to the creation of new defects as well as to spin-activation of pre-existing structural defects (precursors). The activated fraction of defects saturates at a certain dose level depending on the amount of precursors present in the quartz, whereas the newly created defects increase linearly with dose.

Several limitations of the present study should be pointed out.

This study does not explicitly address possible dependencies of the dosimeter response on beam energy and dose rate. Regarding dose rate, no significant dependency has been reported in the literature for various types of quartz and irradiation beams [31], [51], [52]. The beam energy dependence for MV photon irradiation is an open question and needs further systematic investigations. However, good agreement of the dose distributions obtained via EPRI in comparison with the respective MC-simulation is shown in Figure 8. The external magnetic field of $1 \mathrm{~T}$ leads to a considerable inhomogeneity in dose as well as in the energy spectrum of the secondary electrons across the quartz tube. It may therefore be concluded indirectly that both dependencies are minor for the beam energy and dose rate levels investigated in this work.

The presented dose distributions may slightly differ from the dose distribution in real tissue surrounding the air cavity. Primarily due to its density of about $2.2 \mathrm{~g} / \mathrm{cm}^{3}$ fused quartz is lacking water equivalence. 
However, the presented EPRI method is not restricted to fused quartz samples but applicable to future sample materials meeting the call for water equivalence and simultaneously exhibiting appropriate characteristics for EPRI.

Different detection techniques are applicable for EPRI [28], [53]. High spatial resolutions down to the micron-scale have been demonstrated by applying electron spin resonance microscopy to solid samples [54]. With the setup and detection method used in this work, spatial resolutions of approximately $100 \mu \mathrm{m}$ were obtained. Given the gradient strength of $150 \mathrm{G} / \mathrm{cm}$ and the quartz absorption spectrum, a maximum resolution of approximately $53 \mu \mathrm{m}$ is theoretically achievable, but the actual imaging resolution also depends on the SNR. Relatively high doses (600 Gy) and a measuring time of 24 hours were necessary to obtain a sufficient SNR for image reconstruction (Figure 5).

Future research may address different sample geometries and materials as well as different EPR imaging techniques like Rapid Scan EPR Imaging [44], [55] in order to enhance the detection sensitivity per unit time. An increased SNR per unit time as well as reduced irradiation doses and measurement times could thereby be achieved.

Due to their small size, EPRI dosimeters may be inserted into anthropomorphic phantoms. Thus, more complex and realistic MRI guided IMRT treatments'may be investigated.

In this context, EPRI may serve as a future quality assurance tool for the verification of clinical dose calculation algorithms in small volumes, especially at tissue-air/lung interfaces. Typical dose grids applied in treatment planning systems have spatial resolutions in the order of millimeters. In case of lower spatial resolution requirements, the established experimental procedure can be performed faster and with lower irradiation doses.

In addition, EPRI can be performed in three spatial dimensions [56] and may thus serve as a volumetric dosimetry tool. 
S Höfel et al.

Manuscript submitted to Physics in Medicine and Biology

\section{Conclusion}

In this work, EPRI was applied for the first time to quantify the ERE. The ERE was investiagted within a sub-millimeter surface layer surrounding millimeter-size air cavities (I.D. of 3 or $4 \mathrm{~mm}$ ).

The results provide experimental evidence for considerable dosimetric effects due to a transverse, magnetic field of $1.0 \mathrm{~T}$. Local dose enhancements (up to $28 \%$ ) and reductions (up to $35 \%$ ) were measured. The results further indicate that for millimeter-size air cavities the dosimetric impact of the magnetic field on the surface dose distribution around the air cavity increases with increasing cavity size.

The experimentally derived dose distributions are quantitatively in good agreement (passrate of $96 \%$ (TUB4) and $92 \%$ (TUB3) for a $3 \% / 10^{\circ}$ global gamma criterion) with the corresponding MC simulation results. These findings confirm the feasibility of the experimental procedure.

EPRI may play a future role for the investigation of dose distributions within sub-millimeter surface layers at tissue-air interfaces and may thus serve as a complementary experimental tool for relative dosimetry in the presence of external magnetic fields (MRgRT).

\section{Acknowledgements}

The authors gratefully acknowledge the help of Dr. Daniel Frei from Inselspital in Bern regarding the implementation of the phase-space file into the Geant4 MC simulation code. The authors further would like to thank the group members Dr. Mikhailo Azarkh and Dr. Martin Spitzbarth for their training regarding the setup of the EPR experiments and the handling of the image reconstruction software. We also thank Martin v. Bischopinck for measuring the resonator's profile during his bachelor project.

All authors disclose any potential conflict of interest. Edmond Sterpin was partially supported by Accuray for the simulation of the TomoTherapy machine. 


\section{References}

[1] Nutting C, Dearnaley D P and Webb S 2000 Intensity modulated radiation therapy: a clinical review. BJR 73 459-69

[2] Jaffray D A 2012 Image-guided radiotherapy: from current concept to future perspectives Nature Reviews Clinical Oncology 9 688-99

[3] Dempsey J F, Benoit D, Fitzsimmons J R, Haghighat A, Li J G, Low D A, Sjoden G E 2005 A Device for Realtime 3D Image-Guided IMRT International Journal of Radiation Oncology * Biology * Physics 63202

[4] Raaymakers B W et al. 2009 Integrating a 1.5 T MRI scanner with a 6 MV accelerator: proof of concept Phys. Med. Biol. 54229

-[5] Fallone B G 2014 The Rotating Biplanar Linac-Magnetic Resonance Imaging System Seminars in Radiation Oncology 24 200-2

[6] Oborn B M, Kolling S, Metcalfe P E, Crozier S, Litzenberg D W, and Keall P J 2014 Electron contamination modelling and reduction in a 1 T open bore inline MRI-linac system Medical Physics 41051708

[7] Raaymakers B W et al. 2017 First patients treated with a 1.5 TMRI-Linac: clinical proof of concept of a high-precision, high-field MRI guided radiotherapy treatment Phys. Med. Biol. 6223

[8] Mutic S and Dempsey J F 2014 The ViewRay System: Magnetic Resonance-Guided and Controlled Radiotherapy Seminars in Radiation Oncology 24 196-199

[9] Raaymakers B W, Raaijmakers A J E, Kotte A N T J, Jette D and Lagendijk J J W 2004 Integrating a MRI scanner with a $6 \mathrm{MV}$ radiotherapy accelerator: dose deposition in a transverse magnetic field Phys. Med. Biol. 494109

[10] Raaijmakers A J E, Raaymakers B W and Lagendijk J J W 2005 Integrating a MRI scanner with a 6 MV radiotherapy accelerator: dose increase at tissue-air interfaces in a lateral magnetic field due to returning electrons Phys. Med. Biol. 501363

[11] Richter S, Pojtinger S, Mönnich D, Dohm O S and Thorwarth D 2017 Influence of a transverse magnetic field on the dose deposited by a $6 \mathrm{MV}$ linear accelerator Current Directions in Biomedical Engineering 3281

[12] Rubinstein A E, Gay S, Peterson C B, Kingsley C V, Tailor R C, Pollard-Larkin J M, Melancon A D, Followill D S and Court L E 2018 Radiation-induced lung toxicity in mice irradiated in a strong magnetic fièld PLOS ONE 13 e0205803

[13] Mahdavi S R, Esmaeeli A D, Pouladian M, Monfared A S, Sardari D and Bagheri S 2015 Breast dosimetry in transverse and longitudinal field MRI-Linac radiotherapy systems Medical Physics $\mathbf{4 2}$ 925-36 
[14] Oborn B M, Metcalfe P E, Butson M J and Rosenfeld A B 2009 High resolution entry and exit Monte Carlo dose calculations from a linear accelerator $6 \mathrm{MV}$ beam under the influence of transverse magnetic fields Medical Physics 36 3549-59

[15] Oborn B M, Metcalfe P E, Butson M J and Rosenfeld A B 2010 Monte Carlo characterization of skin doses in $6 \mathrm{MV}$ transverse field MRI-linac systems: Effect of field size, surface orientation, magnetic field strength, and exit bolus Medical Physics 37 5208-17

[16] Agostinelli S et al. 2003 Geant4 - a simulation toolkit Nuclear Instruments and Methods in Physics Research Section A: Accelerators, Spectrometers, Detectors and Associated Equipment, 5063250

[17] Carrier J-F, Archambault L, Beaulieu L and Roy R 2004 Validation of GEANT4, an object-oriented Monte Carlo toolkit, for simulations in medical physics Medical Physics 31 484-92

[18] Raaijmakers A J E, Raaymakers B W and Lagendijk J J W 2007 Experimental verification of magnetic field dose effects for the MRI-accelerator Phys. Med. Biol. 524283

[19] Ahmad S B, Sarfehnia A, Paudel M R, Kim A, Hissoiny S, Sahgal A and Keller B 2016 Evaluation of a commercial MRI Linac based Monte Carlo dose calculation algorithm with geant 4 Medical Physics 43 894-907

[20] Delfs B, Schoenfeld A A, Poppinga D, Kapsch R-P, Jiang P, Harder D, Poppe B and Looe H K 2017 Magnetic fields are causing small, but significant changes of the radiochromic EBT3 film response to $6 \mathrm{MV}$ photons Phys. Med. Biol. 63035028

[21] Lee H J, Roed Y, Venkataraman S, Carroll M and Ibbott G S 2017 Investigation of magnetic field effects on the dose-response of 3D dosimeters for magnetic resonance - image guided radiation therapy applications Radiotherapy and Oncology 125 426-432

[22] Lee H J, Kadbi M, Bosco G and Ibbott G S 2018 Real-time volumetric relative dosimetry for magnetic resonance - image-guided radiation therapy (MR-IGRT) Phys. Med. Biol. 63045021

[23] Costa F, Doran S J, Hanson I M, Nill S, Billas I, Shipley D R, Duane S, Adamovics J and Oelfke U 2018 Investigating the effect of a magnetic field on dose distributions at phantom-air interfaces using PRESAGE ${ }^{\circledR}$ 3D dosimeter and Monte Carlo simulations Phys. Med. Biol. 6305 NT01

[24] Lee H J, Choi G W, Alqathami M, Kadbi M and Ibbott G 2017 Using 3D dosimetry to quantify the Electron Return Effect (ERE) for MR-image-guided radiation therapy (MR-IGRT) applications J. Phys.: Conf. Ser. 847012057

[25] Avanzo M, Rink A, Dassie A, Massarut S, Roncadin M, Borsatti E and Capra E 2012 In vivo dosimetry with radiochromic films in low-voltage intraoperative radiotherapy of the breast Medical Physics $392359-68$ 
[26] Yu P K N, Butson M and Cheung T 2006 Does mechanical pressure on radiochromic film affect optical absorption and dosimetry? Australas. Phys. Eng. Sci. Med. 29 285-7

[27] Lund A and Shiotani M 2014 Applications of EPR in Radiation Research (Springer International Publishing)

[28] Eaton G R and Eaton S S 1995 Introduction to EPR imaging using magnetic-field gradients Concepts Magn. Reson. 7 49-67

[29] Eaton G R and Eaton S S 1986 Electron spin-echo-detected EPR imaging Journal of Magnetíc Resonance (1969) 67 73-7

[30] León M, Martín P, Ibarra A and Hodgson E R 2009 Gamma irradiation induced defeçts in different types of fused silica Journal of Nuclear Materials 386-388 1034-7

[31] Ziaie F, Mohammadzadeh A and Modarresi M 2009 Stability study of free radicals in $10 \mathrm{MeV}$ electron beam irradiated quartz as an EPR dosimetry method Nukleonika 54 251-4

[32] Agnello S, Boscaino R, Cannas M and Gelardi F M 2001 Instantaneous diffusion effect on spin-echo decay: Experimental investigation by spectral selective excitation Phys. Rev. B 64174423

[33] Weeks R A 1956 Paramagnetic Resonance of Lattice Defects in Irradiated Quartz Journal of Applied Physics 27 1376-81

[34] Silsbee R H 1961 Electron Spin Resonance in Neutron-Irradiated Quartz Journal of Applied Physics 32 1459-62

[35] Yip K L and Fowler W B 1975 Electronic structure of E' centers in SiO2 Phys. Rev. B 11 2327-38

[36] Feigl F J and Anderson J H 1970 Defects in crystalline quartz: Electron paramagnetic resonance of E' vacancy centers associated with germanium impurities Journal of Physics and Chemistry of Solids 31 575-96

[37] Stapelbroek M, Griscom D L, Friebele E J and Sigel G H 1979 Oxygen-associated trapped-hole centers in high-purity fused silicas Journal of Non-Crystalline Solids 32 313-26

[38] Friebele E J, Griscom D L, Stapelbroek M and Weeks R A 1979 Fundamental Defect Centers in Glass: The Peroxy Radical in Irradiated, High-Purity, Fused Silica Phys. Rev. Lett. 42 1346-9

[39] Galeener F L, Kerwin D B, Miller A J and Mikkelsen J C 1993 X-ray creation and activation of electron spin resonance in vitreous silica Phys. Rev. B 47 7760-79

[40] Spitzbarth $M$ and Drescher M 2015 Simultaneous iterative reconstruction technique software for spectral-spatial EPR imaging Journal of Magnetic Resonance 257 79-88

[41] Lee J, Lee J, Ryu D, Lee H and Ye S-J 2018 Fano cavity test for electron Monte Carlo transport algorithms in magnetic fields: comparison between EGSnrc, PENELOPE, MCNP6 and Geant4 Phys. Med. Biol. 63195013 
[42] Sterpin E, Tomsej M, Cravens B, Salvat F, Ruchala K, Olivera G H and Vynckier S 2007 Monte Carlo simulation of the Tomotherapy treatment unit in the static mode using MC HAMMER, a Monte Carlo tool dedicated to Tomotherapy J. Phys.: Conf. Ser. 74021019

[43] Sterpin E, Salvat F, Cravens R, Ruchala K, Olivera G H and Vynckier S 2008 Monte Carlo simulation of helical tomotherapy with PENELOPE Phys. Med. Biol. 53 2161-2180

[44] Mitchell D G, Quine R W, Tseitlin M, Meyer V, Eaton S S and Eaton G R 2011 Comparison of Continuous Wave, Spin Echo, and Rapid Scan EPR of Irradiated Fused Quartz Radiat. Meas. 46 993-6

[45] Low D A, Harms W B, Mutic S and Purdy J A 1998 A technique for the quantitative evaluation of dose distributions Medical Physics 25 656-61

[46] Levêque P, Godechal Q, Bol A, Trompier F and Gallez B 2009 X-band EPR imaging as a tool for gradient dose reconstruction in irradiated bones Med. Phys. 36 4223-9

[47] Vanea E S, Levêque P, Abboud F, Bol A, Denis J M, Kolbun N, Vynckier S and Gallez B 2009 Evaluation of the dose distribution gradient in the close vicinity of brachytherapy seeds using electron paramagnetic resonance imaging Magn. Reson. Med. 61 1225-31

[48] Kolbun N, Adolfsson E, Gustafsson H and Lund E 2014 High-resolution mapping of 1D and 2D dose distributions using X-band electron paramagnetic resonance imaging Radiat. Prot. Dosim. 159 $182-7$

[49] Agnello S, Boscaino R, Buscarino G, Cannas M and Gelardi F M 2002 Structural relaxation of E' centers in amorphous silica Phys. Rev. B 66113201

[50] Griscom D L 1984 Characterization of three $E^{\prime}$-center variants in X-and $\mathrm{Y}$-irradiated high purity a$\mathrm{SiO} 2$ Nuclear Instruments and Methods in Physics Research Section B: Beam Interactions with Materials and Atoms 1 481-8

[51] Mashkov V A, Austin W R, Zhang $L$ and Leisure R G 1996 Fundamental Role of Creation and Activation in Radiation-Induced Defect Production in High-Purity Amorphous SiO2 Phys. Rev. Lett. 76 2926-9

[52] Pfeffer R L 1985 Damage center formation in SiO2 thin films by fast electron irradiation Journal of Applied Physics 57 5176-80

[53] Atherton N M, Davies M J, Gilbert B C, Eaton S S and Eaton G R 1996 EPR imaging Electron Spin Resonance pp 169-85

[54] Blank A, Suhovoy E, Halevy R, Shtirberg L and Harneit W 2009 ESR imaging in solid phase down to sub-micron resolution: methodology and applications Physical Chemistry Chemical Physics 11 6689-99 
[55] Eaton S S, Shi Y, Woodcock L, Buchanan L A, McPeak J, Quine R W, Rinard G A, Epel B, Halpern H J and Eaton G R 2017 Rapid-scan EPR imaging Journal of Magnetic Resonance 280 140-8

[56] Deng Y, Petryakov S, He G, Kesselring E, Kuppusamy P and Zweier J L 2007 Fast 3D spatial EPR imaging using spiral magnetic field gradient Journal of Magnetic Resonance 185 283-90 